\title{
Adult onset Still's disease: a comparison of two clinical cases
}

\author{
Livio Bonsignore, ${ }^{1}$ Roberto Cardillo, ${ }^{1}$ Salvatore Leotta, ${ }^{1}$ Sebastiano Pavone, ${ }^{1}$ Alfonsa Lucia Dimora, ${ }^{1}$ \\ Irene Alfina Cavallaro, ${ }^{1}$ Rosaria Platania, ${ }^{1}$ Nunzio Maurizio Catania, ${ }^{2}$ Rocco Siciliano ${ }^{1}$ \\ ${ }^{1}$ UOC di Medicina Interna, PO S. Marta e S. Venera; ${ }^{2}$ Patologia Clinica, PO S. Marta e S. Venera, Acireale (CT), Italy
}

\begin{abstract}
Still's disease is a disease of unknown etiology that was identified for the first time in 1897 by George Still who noted the association of fever, arthralgias and cutaneous rash in a group of 22 children. In 1971, Bywaters noticed that this symptomatology could also be found in adult patients. High fever, arthralgias, a diffuse cutaneous rash with sore throat, increased spleen volume and lymph nodes are the clinical manifestations of this disease. However, it shows high variability in its clinical presentation (monocyclic, polycyclic and chronic forms). Blood tests show high levels of white blood cells, increased ferritin levels, and negative autoantibodies and rheumatoid factor. We examined 2 clinical cases that strongly suggested adult Still's disease: a 38year old woman and a 30-year old man. The woman came to our attention because of the following symptomatology: fever for more than eleven days before hospitalization and polyarthralgias. Blood tests showed high inflammatory markers (ferritin $>35,000)$, low platelets and increased white blood cells. A diffuse rash and oral aphthae were then observed. The second case presented the following symptoms: fever $\left(38.4^{\circ} \mathrm{C}\right)$, cutaneous rash, polyarthralgias, and sore throat. Blood tests showed high levels of inflammatory markers and blood cell count showed an increase in neutrophil levels. Abdominal ultrasonography showed hepatosplenomegaly. Concentrating on these elements allowed us to formulate the diagnosis of adult Still's disease, both patients showing a highly suggestive clinical profile. Yamaguchi's criteria were satisfied and different diseases could be excluded according to the pharmacological response to anti-inflammatory drugs. This strengthened our diagnostic hypothesis and allowed us to focus our attention on this unknown disease, often unrecognized because of the problems of diagnosis. In fact, the clinical characters are initially aspecific (sore throat and fever). Furthermore, there is a large inter-individual variability during the disease course that could lead to misunderstanding. However, satisfaction of Yamagouchi's criteria and the pharmacological response to corticosteroid drugs suggested adult Still's disease.
\end{abstract}

\section{Introduction}

Still's disease is a disease of unknown etiology that was identified for the first time in 1897 by George Still who noted the association of fever, arthralgias and cutaneous rash in a group of 22 children. The pathology was later defined as systemic-onset juvenile idiopathic arthritis. In 1971, Bywaters noticed that febrile polyarthritis with similar characteristics to the systemic form of chronic juvenile arthritis could also be found

Correspondence: Livio Bonsignore, via Caronia, Acireale (CT), Italy. Tel. +39.348.3133566.

E-mail: libonsi@alice.it

Key words: Still's disease, adult onset, fever, arthralgias, cutaneous rash.

Conflict of interests: the authors declare no potential conflict of interests.

This work is licensed under a Creative Commons Attribution NonCommercial 3.0 License (CC BY-NC 3.0).

CCopyright L. Bonsignore et al., 2013

Licensee PAGEPress, Italy

Italian Journal of Medicine 2013; 7:310-314

doi:10.4081/itjm.2013.310 in adult patients. In reality, a similar if not identical presentation to that of adult Still's disease with associated joint involvement had already been described under the name allergic-hyperergic subsepsis or Wissler-Fanconi syndrome. The similarity between these two conditions was so evident that in 1973 Cairot et al. concluded that it was probably the same disease.

Onset is usually acute, characterized by high temperatures, arthralgias, macular or maculopapular rash to the body, the face, the lower limbs, often coinciding with episodes of fever, pharyngodynia, lymphodenopathy and/or splenomegaly (Table 1)..$^{1,2}$ Laboratory tests reveal neutrophil leukocytosis, increase in aspecific inflammatory indexes, and negative rheumatoid factor and antinuclear antibodies (Table 2).

The clinical course is extremely variable and unpredictable. Nevertheless, it can be divided into three main patterns: i) a monocyclic form that consists of a single episode; ii) a polycyclic form of intermittent character with periods of complete remission alternating with periods of representation; and iii) a chronic form characterized by a persistent disease activity with erosive arthritis. ${ }^{1}$ Since 1971, the year in which Bywaters described the first cases, the number of studies dedicated to this pathology has progressively grown. But in spite of this, the clinical cases described in the literature only amount to a few hundred. 
The clinical profile is often unclear and it is difficult to make a reliable diagnosis. This has probably led to an underevaluation of the disease incidence. Table 3 shows a summary of inclusion and exclusion criteria.

\section{Case Report \#1}

A 38-year old woman, with an uneventful case history until 11 days before admission, had an onset of fever with shivers and widespread arthralgia. Her family doctor prescribed antibiotics with macrolides that the patient had to interrupt because of onset of vomiting. Blood tests showed an increase in aspecific inflammatory indexes among which high ferritin values $(>35,000)$ and a big increase in transaminase (Table 4). Eleven days after the onset of symptoms the patient went to the Emergency Department and was sent on to our department.

Objective examination showed liver extending 2 $\mathrm{cm}$ beyond the costal arch and a slight splenomegaly. There were no pathological findings involving the respiratory or cardiac apparatus, and neither were there any cutaneous or mucosal alterations. Body temperature on admission was $38^{\circ} \mathrm{C}$.

Blood tests taken on admission in our unit confirmed an increase in transaminase and showed modest neutrophil leukocytosis and piastrinopenia. There was a large increase in ferritin levels (although with values below those observed before admission) and an increase in lactate dehydrogenase (LDH). Echography of the upper abdomen showed modest hepatosplenomegaly.

Since a precise profile had still not been confirmed, we started empirical antibiotic treatment with quinolones (ciprofloxacin 1 capsule $\times 2 /$ die) and tienam $500 \mathrm{mg} \times 2$ endovenous (e.v.) was administered the day after. Since fever persisted, e.v. methylprednisolone was added at a dose of $20 \mathrm{mg} \times 2$; fever symptoms disappeared on Day 2 of steroid treatment. Therefore, the antibiotic treatment was suspended, and after four days the steroid treatment was reduced to $20 \mathrm{mg}$ of methylprednisolone in a single administration in the morning with a scaled reduction in dose.

On Day 9 after admission, the patient presented erythematous rash on the front of the chest and abdomen, and night-time body temperature of around $40^{\circ} \mathrm{C}$ with general malaise and a renewed presentation of acute erythematous manifestations. Steroid therapy was once again increased (e.v. methylprednisolone 40 $\mathrm{mg}$ in a single administration); the fever and the esantematic manifestations both disappeared after approximately eight days.

On being discharged from the hospital, the patient presented a big reduction in erythrocyte sedimentation rate (ESR) on polymerase chain reaction (PCR), and a return to normal ferritin and LDH values. Blood tests showed normocytic anemia with normal platelet count.

At the 1-year follow up, the patient presented neither fever nor erythematous manifestations.

The patient consented to the publication of her case report.

\section{Case Report \#2}

A hypertense and obese 30-year old man came to our attention with one week of fever, cutaneous rash mainly on the body, polyarthritis and pharyngodynia.

Table 1. Main clinical symptoms of Still's disease.

\begin{tabular}{lc}
\hline Arthralgias & \% of cases \\
\hline Fever $>39^{\circ} \mathrm{C}$ & 100 \\
\hline Cutaneous rash & 96 \\
\hline Pharyngodynia & 88 \\
\hline Lymphodenopathy & 70 \\
\hline Splenomegaly & 63 \\
\hline
\end{tabular}

Table 2. Still's disease: main laboratory data.

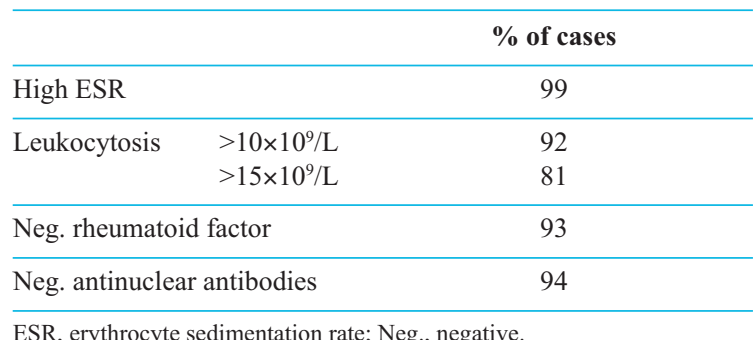

Table 3. Diagnostic criteria for adult Still's disease.

Main inclusion criteria

Fever $\left(>39^{\circ} \mathrm{C}\right)$ for $>$ week

Arthralgias of at least 2 weeks

Cutaneous rash

Leukocytosis $\left(>10 \times 10^{9} / \mathrm{L}\right)$

Minor criteria

Pharyngodynia

Lymphodenopathy and/or splenomegaly

Neg. rheumatoid factor

Neg. antinuclear antibodies

Exclusion criteria

Infections

Neoplasias

Rheumatic disease

Neg., negative. Modified from Yamaguchi et al.,1992.2 
His family doctor had prescribed oral cephalosporin and antihistamines but with little benefit.

Given that symptoms persisted, the patient came to the Emergency Department and neutrophil leukocytosis was confirmed. The patient was admitted to our department with clear erythematous blotches to the body and hepatomegaly was observed (liver extending $2 \mathrm{~cm}$ from the costal arch) and slight splenomegaly. The patient also complained of intense polyarthralgia together with the appearance of clearly defined erythematous manifestations and pharyngodynia. Chest examination showed no pathological involvement.
Blood tests carried out on admission showed an increase in ESR, PCR, ferritin, fibrinogen, neutrophil leukocytosis, and an increase in $\gamma$-GT (Table 5). Blood culture was normal, as were Widal-Wright and WeilFelix serum tests, and autoimmunity tests (ANA, ASMA, n-DNA, c-ANCA, p-ANCA and anti-ENA).

Abdominal echography showed moderate hepatic steatosis and modest hepatosplenomegaly.

Echocardiogram showed no changes worthy of note.

Given the hypothesis of Still's disease, treatment was started with $25 \mathrm{mg}$ prednisone twice a day and a reduction in symptoms was already seen on Day 3

Table 4. Case 1: hematochemical tests.

\begin{tabular}{llcc}
\hline & & On admission & On discharge \\
\hline RBC & $\times 10^{12} / \mathrm{L}$ & $3,500,000$ & $3,700,000$ \\
\hline $\mathrm{WBC}$ & $\times 10^{9} / \mathrm{L}$ & 16 & 8.3 \\
\hline Hemoglobin & $\mathrm{g} / \mathrm{dL}$ & 11.6 & 11 \\
\hline PLT & $\times 10^{9} / \mathrm{L}$ & 50 & 137 \\
\hline LDH & $\mathrm{u} / \mathrm{L}$ & 780 & 420 \\
\hline ESR & $\mathrm{mm} / \mathrm{h}$ & 44 & 22 \\
\hline CRP & & 84 & 40 \\
\hline GOT & & 200 & 39 \\
\hline GPT & & 150 & 23 \\
\hline Ferritin & 5040 & 390 \\
\hline Sideremia & & 33 & - \\
\hline
\end{tabular}

RBC, red blood cell count; WBC, white blood cell count; PLT, platelet count; LDH, lactate dehydrogenase; ESR, erythrocyte sedimentation rate; CRP, C-reactive protein; GOT, glutamic-oxaloacetic transaminase; GPT, glutamic-pyruvic transaminase.

Table 5. Case 2: hematochemical tests.

\begin{tabular}{llcc}
\hline & & On admission & On discharge \\
\hline RBC & $\times 10^{12} / \mathrm{L}$ & 5180 & 13,500 \\
\hline $\mathrm{WBC}$ & $\times 10^{9} / \mathrm{L}$ & 18,000 & 4900 \\
\hline Hemoglobin & $\mathrm{g} / \mathrm{dL}$ & 14 & 14 \\
\hline PLT & $\times 10^{9} / \mathrm{L}$ & 254 & 3330 \\
\hline $\mathrm{LDH}$ & $\mathrm{u} / \mathrm{L}$ & 400 & - \\
\hline ESR & $\mathrm{mm} / \mathrm{h}$ & 80 & 40 \\
\hline CRP & & 96 & 26 \\
\hline GOT & & 23 & 26 \\
\hline GPT & 39 & 30 \\
\hline Ferritin & 1530 & 598 \\
\hline Sideremia & 20 & - \\
\hline Fibrinogen & 1020 & 583 \\
\hline RBC, red blood cell count; WBC, white blood cell count; PLT, platelet count; LDH, lactate dehydrogenase; ESR, erythrocyte sedimentation rate; CRP, C-reactive \\
protein; GOT, glutamic-oxaloacetic transaminase; GPT, glutamic-pyruvic transaminase.
\end{tabular}


with disappearance of fever and normalization of leukocyte count. Cortisone therapy was continued until discharge and a dose of $25 \mathrm{mg} /$ die was prescribed to be taken at home together with $500 \mathrm{mg} / \mathrm{die}$ naproxen for 20 days to act in synergy with the steroid to better control the arthralgias. Regular periodic clinical and biohumoral checkups were programmed and these showed a return to normal of all the altered biohumoral parameters and a complete remission of symptoms at three months with 5 mg prednisone.

The patient consented to the publication of his case report.

\section{Discussion}

Still's disease in adults is a rare pathology that seems to have a greater frequency in females before the age of 35 years. However, in recent years, cases of Still's disease have been reported with a later onset. Our first case history regards a 38-year old patient whose clinical profile was characterized by widespread arthralgia accompanied by fever that did not respond to antibiotics. Not all laboratory data were suggestive of Still's disease, in spite of presentation of normocytic anemia characteristic of chronic inflammatory disease and marked piastrinopenia that suggested chronic inflammatory disease (CID). In fact, the presence of CID in adult Still's disease has been described by some Authors. ${ }^{3,4}$ Laboratory data also showed an increase in the aspecific inflammatory indexes, and a moderate increase in transaminase values, $\gamma$-GT and alkaline phosphatase. ${ }^{5,6}$

Objective abdominal examination showed only a modest hepatomegaly. Tests for infectious or neoplastic diseases were all negative. Autoimmune diseases (vasculitis, systemic lupus erythematosus, rheumatoid arthritis) were excluded one by one by the lack of other symptoms and after negative laboratory and instrumental tests. The big increase in ferritin levels was important for diagnosis.

Hyperferritinemia was also reported in other cases in the literature ${ }^{7}$ and, in spite of the fact that this was not included in the diagnostic criteria, some Authors used this parameter not only to reinforce the diagnosis but also to monitor disease course over time and to evaluate response to therapy. In addition, hyperferritinemia together with the other data (alteration in the hepatic function indexes, hepatosplenomegaly) is a laboratory parameter for macrophage activation syndrome. This is a potentially fatal complication of rheumatic diseases that is most frequently seen in juvenile idiopathic arthritis and in adult Still's disease..$^{7-9}$ It is characterized by pancytopenia, hepatic deficiency, coagulopathy and neurological symptoms, and is considered secondary to the activation and uncontrolled proliferation of $\mathrm{T}$ lymphocytes and macrophages that determine diffuse hemophagocytosis and hyperproduction of cytokines. It was, therefore, hypothesized that this could be a case of adult Still's disease with initial macrophage activation syndrome. Steroid therapy was started that led to the disappearance of the fever, of the arthralgias and normalization of the aspecific inflammatory indexes and ferritin levels. The absence of any representation of fever, arthralgias and erythematous rash one year after suspension of therapy suggested a monocyclic form of the disease.

The symptoms presented in our second case, together with laboratory data, led us to consider the possibility that this was a presentation of Still's disease. Yamaguchi's diagnostic criteria for the disease are: neutrophil leukocytosis, polyarthralgia, cutaneous rash, high fever, pharyngodynia, negative for antibodies, lymphadenopathy and/or splenomegaly, increase in transaminase and LDH levels (Table 3). In this case, if we do not consider the renewed rise in transaminase during hospitalization, the criteria listed here were all present. On the other hand, the fact that hepatic enzyme values were normal during hospitalization does not mean we can exclude the possibility that these could have been altered in a previous phase, even more so since objective examination and abdominal echography both showed hepatomegaly. Therefore, there was hepatic involvement, albeit in a mild form. For these reasons, in this case, we can reasonably presume that the diagnosis is one of adult Still's disease.

\section{Conclusions}

Even today, adult Still's disease is a clinical entity that remains to be clearly defined, above all with regards to the different ways in which the disease evolves during its course. ${ }^{1}$ Among these, the monocyclic type (that only runs through a single episode) certainly raises the most doubts as to whether one is dealing with a disease or a syndrome. Furthermore, presentation of fever in a young adult (sometimes even a high fever), accompanied by intense painful polyarticular symptoms with stiffness and a sore throat, above all if associated with a cutaneous rash and neutrophil leukocytosis, must raise suspicion of Still's disease. In these cases, even in the absence of organ involvement, a marked increase in ferritin levels should lead the physician to consider Still's disease and to verify Yamaguchi's diagnostic criteria. ${ }^{10}$

The difference between our 2 cases consists in the association with the macrophage activation syndrome and the monocyclic disease course of Case \#1 (without, therefore, further cortisone treatment) while the patient in Case \#2 has been under cortisone treatment for a year, even though some satisfactory benefit 
has been achieved. These 2 cases are, therefore, examples of the polymorphic nature of adult Still's disease.

\section{References}

1. Fietta P, Manganelli P. Adult onset Still's disease. Minerva Med 2002;93:27-39.

2. Yamaguchi M, Ohta A, Tsunematsu T. Preliminary criteria for classification of adult Still's disease. J Rheumatol 1992;19:424-30.

3. Park JH, Bae JH, Choi YS, et al. Adult-onset Still's disease with disseminated intravascular coagulation and multiple organ dysfunctions dramatically treated with cyclosporine A. J Korean Med Sci 2004;19:137-41.

4. Yokoyama M, Suwa A, Shinozawa T, et al. A case of adult onset Still's disease complicated with adult respiratory distress syndrome and disseminated intravascular coagulation. Nihon Rinsho Meneki Gakkai 1995;18: 207-14
5. Kirino Y, Takeno M, Iwasaki M, et al. Increased serum HO-1 in hemophagocytic syndrome and adult-onset Still's disease: use in the differential diagnosis of hyperferritinemia. Arthritis Res Ther 2005;7:616-24.

6. Andrès E, Kurtz JE, Perrin AE, et al. Retrospective monocentric study of 17 patients with adult Still's disease, with special focus on liver abnormalities. Hepatogastroenterology 2003;50:192-5.

7. Chahine B, Luthier F. Value of hyperferritinemia and glycosylated ferritin in the diagnosis of adult-onset Still's disease. 3 case reports. Press Med 2005;34: 928-32.

8. Fietta P, Manganelli P. The hemophagocytic syndrome (macrophage activation syndrome). Minerva Med 2003;94:19-27.

9. Hamidou M, Boutoille D, Masseau A, et al. Adult-onset Still disease with hemophagocytic syndrome treated with cyclosporine. Presse Med 2005;34:1634-6.

10. Govoni M, Ivorra V, Rizzo N, Trotta F. La malattia di Still dell'adulto. Atti XXXVI Congresso Nazionale SIR 1999. Reumatismo 1999;51:173-6. 